\section{Rheopexy in Dilute Polymer Solutions at Low Shear Gradients}

ONE of us has pointed out that theoretical consideration of the hydrodynamic interaction of a very long flexible chain in laminar flow suggests that under certain conditions an increaso in the viscosity of a polymer solution may occur with increase in the shear gradient ${ }^{1}$. Such rheopexy should be favoured with a solution of a linear polymer of very high molecular woight in an extremely viscous solvent, otherwise the theory predicts only a decrease in solution viscosity with increasing gradient such as has been reported experimentally in numerous instances. Generally, examples of rheopexy reported for polymer solutions occur at high gradients and reasonably have been attributed to the onset of turbulent flow, but this would not provide a likely explanation of an increase in viscosity reported by Selby and Hunstad which begins at only about $100 \mathrm{sec}^{-1}$. The latter results are consistent with the theory mentioned here inasmuch as the system studied was a polymethylmethacrylate of high molecular weight $\left(\sim 10^{6}\right)$ dissolved in a mineral oil with a viscosity of several hundred poise but are not directly apposite because of the large concentration of polymer ( 3 por cent) ${ }^{2}$. A direct test of the theory has been undertaken by Wolff, who reported viscosity data extrapolated to infinite dilution for solutions of polystyrene ( $\mathrm{M}$ up to $\left.10^{7}\right)$ in a chlorinated diphenyl $(\eta \sim 1$ poise), with the conclusion that no increase in viscosity occurred ${ }^{3}$. The present communication reports further preliminary experiments designed to test the theory.

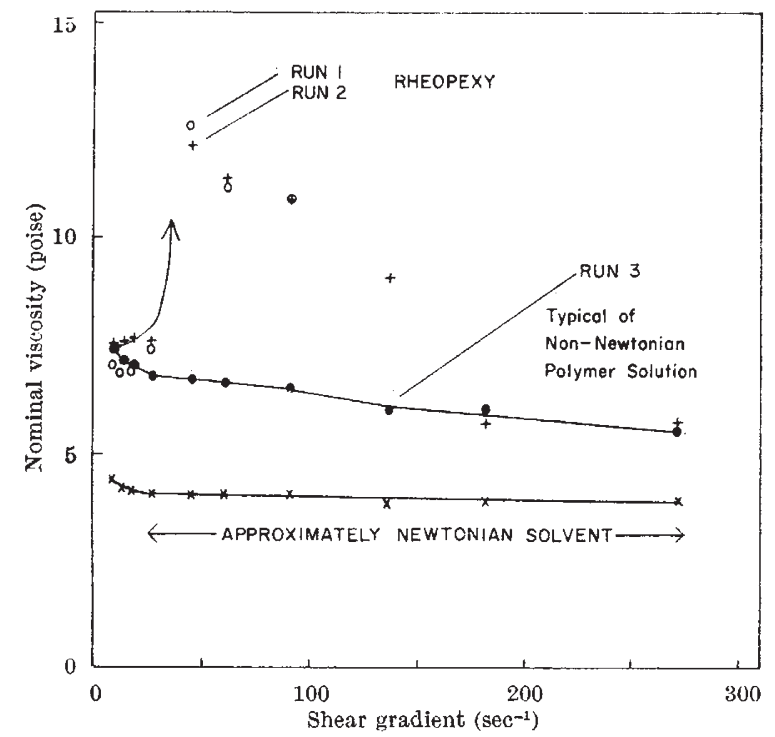

Fig. 1. Dependence of viscosity on shear gradient. $x$, Chlorinated
diphenyl; $O,+, 0$, solution of polymethylmethacrylate in chlorinated diphenyl

Polymethylmethacrylates, polymorized by conventional radical polymerization, of $\mathrm{M}_{\mathrm{W}} \sim 4.2$ and $1.0 \times 10^{6}$ were dissolved in a chlorinated diphenyl containing 0.2 per cent 4-tert-butylcatechol. Solutions were sheared at an ambient temperature of about $25^{\circ} \mathrm{C}$ in a plate and conc consistometer ${ }^{4}$. The results in Fig. 1 obtained with $2 \times$ $10^{-3} \mathrm{~g} / \mathrm{ml}$. of the higher molecular weight polymer show an increase in nominal viscosity (the absolute calibration of the machine has not yet been checked) at low shear gradients, which is reproducible as a gross effect provided the solution is subjected to only limited shearing (cf. runs 1 and 2). After the more extensive shearing during run 2 plus $1 \mathrm{~h}$ at the highest gradient of $272 \mathrm{sec}^{-1}$ the solution showed only a decrease in viscosity in two further runs (only run 3 is shown). However, when this same solution was cooled to near $0^{\circ} \mathrm{C}$ and its viscosity thereby increased to several hundred poise, marked rheopexy was again detected and could again be eliminated by prolonged shearing. Qualitatively similar results were obtained with the original solution in Cannon-Fenske capillary viscometers. By contrast, a more concentrated solution of the lower molecular weight polymer $\left(5 \times 10^{-3} \mathrm{~g} / \mathrm{ml}\right.$.) showed no rheopexy at ambient temperature but only a decrease in viscosity with increasing gradient of tho typo generally reported for polymer solutions.

These experiments show that rheopexy is definitely observed in these solutions and at such low gradients that turbulent flow seems unlikely and, further, indicate that the occurrence of rheopexy is sensitive to viscosity and the length of the polymer molecules. Moreover, this latter sensitivity may be adduced to explain why rhoopexy is eliminated by shearing with the acceptable postulate that the long polymer molecules are broken by the high shear forces applied. This general picture is consistent with theory but more critical and quantitative tests are necessary. The most important is to test whether, as required by theory, rheopexy can be observed in more dilute solutions. So far we have observed an effect near $0^{\circ} \mathrm{C}$ with concentrations of as little as $0.13 \times 10^{-3} \mathrm{~g} / \mathrm{ml}$., but we are still trying to improve control of important experimental factors such as temperature in order to permit extrapolation of results to infinite dilution.

A. Peterlin

D. T. TURNER *

Camille Dreyfus Laboratory,

Research Triangle Institute,

$$
\text { P.O. Box } 490 \text {, }
$$

Durham, North Carolina.

* Postdoctoral Fellow on leave from the Natural Rubber Producers' Research Association.

${ }^{2}$ Peterlin, A., J. Chem. Phys., 33, 1799 (1960); Makromol. Chem., 44-46. 338 (1961); Kolloid Z., 182, 110 (1962); J. Polymer Sci., 8, 621 (1952). ${ }^{2}$ Selby, T. W., and Hunstad, N. A., Symp. Non-Newtonian Viscosity, ASTM Pub. No. 299, 98 (1962).

${ }^{3}$ Wolff, C., J. Chim. Phys., 59, 413 (1962).

${ }^{4}$ Képès, A., J. Polymer Sci., 22, 409 (1956).

\section{Colorimetric Determination of 3,6-Anhydrogalactose with the Indolyl-3-acetic Acid Reagent}

THE sugar 3,6-anhydrogalactose is a major constituent of tho agaroso fraction of agar, of kappa carrageenin, and of some other polysaccharides from marine alga $\theta^{1}$. This sugar can be determined spectrophotometrically as 5-hydroxymethyl-2-furaldohydo ${ }^{2}$ or by an adaptation of the resorcinol test for ketoses ${ }^{3}$. The rato of dogradation of 3,6-anhydrogalactoso to 5-hydroxymethyl-2-furaldehyde suggests that any method used for tho colorimotric: determination of fructose should bo applicable to 3,6 anhydrogalactose. Steinitz ${ }^{4}$ compared six methods for the dotermination of fructose in body fluids, and favourod tho mothod of Heyrovsky ${ }^{5}$, who used $\beta$-indolylacetic acid as the colour reagent. The present communication describes the use of this reagent for the determination of 3,6-anhydrogalactose.

A standard solution of 3,6-anhydrogalactose was preparod from 3,6-anhydro-I-galactoso diethylmereaptal ${ }^{6}$ Hoyrovsky's proceduro ${ }^{5}$ was followed except that the tube was placed in the thermoregulated bath immodiately aftor tho hydrochloric acid was added. The colourdovelopment curves showod that at $\lambda 530 \mathrm{~m} \mu \mathrm{maximum}$ absorbance occurred in $5 \mathrm{~h}$ at $23.5^{\circ}$; in $1 \mathrm{~h}$ at $37^{\circ}$ and in $7 \mathrm{~min}$ at $80^{\circ} \mathrm{C}$. Because of the rapidity of tho roaction at the highor temperatures, careful attention to the details of the proceduro wore necessary. Beor's law was followed with amounts up to $0.25 \mu \mathrm{M}$ of 3,6-anhydrogalactose. The absorbance of the mercaptal was 7 per cont groater than with the free sugar, presumably because of the mercaptan formed in presonce of the acid. A similar increase in absorbance occurred when ethyl mercaptan was present with fructoso. 